\title{
On the viable two leptoquark model for the $B$-physics anomalies
}

\section{Ilja Doršner*t}

University of Split, Faculty of Electrical Engineering, Mechanical Engineering and Naval Architecture in Split (FESB), Ruđera Boškovića 32, 21000 Split, Croatia

E-mail: dorsnerefesb.hr

I present a viable two leptoquark model based on $S U(5)$ gauge symmetry that addresses the $B$ physics anomalies. The entire low-energy flavour structure of the set-up originates from two $S U(5)$ operators that relate Yukawa couplings of the two leptoquarks. The proposed scenario accommodates all measured lepton flavour universality ratios in $B$-meson decays, is consistent with related flavour observables, and is compatible with direct searches at the LHC. The model is self-consistently perturbative, provides gauge coupling unification, and predicts several yetto-be-measured flavour observables. I also outline prospects for future discoveries of the two leptoquarks at the LHC.

European Physical Society Conference on High Energy Physics - EPS-HEP2019 -

10-17 July, 2019

Ghent, Belgium

* Speaker.

${ }^{\dagger}$ Work in collaboration with D. Bečirević, S. Fajfer, N. Košnik, D. A. Faroughy, and O. Sumensari. 


\section{Introduction}

One of the most popular testing grounds for new physics is the disagreement between experimentally measured and theoretically predicted values for $R_{D^{(*)}}=\mathscr{B}\left(B \rightarrow D^{(*)} \tau \bar{v}\right) / \mathscr{B}\left(B \rightarrow D^{(*)} \ell \bar{v}\right)$, $\ell \in(e, \mu)$, and $R_{K^{(*)}}=\mathscr{B}\left(B \rightarrow K^{(*)} \mu \mu\right) /\left.\mathscr{B}\left(B \rightarrow K^{(*)} e e\right)\right|_{q^{2} \in\left[q_{\min }^{2}, q_{\max }^{2}\right.}$, where it is found that $R_{D^{(*)}}^{\exp }>$ $R_{D^{(*)}}^{\mathrm{SM}}$ and $R_{K^{(*)}}^{\exp }<R_{K^{(*)}}^{\mathrm{SM}}$. For the latest fits of the $R_{D^{(*)}}$ and $R_{K^{(*)}}$ discrepancies see, for example, Refs. [1, 2, 3] and Refs. [4, 5, 6, 7], respectively. Interestingly enough, the scale of new physics, i.e., $\Lambda_{\mathrm{NP}}$, that is associated with these two sets of departures from the values predicted within the Standard Model (SM) framework appears not to be one and the same. In particular, if one assumes $\mathscr{O}(1)$ prefactors of the $d=6$ operators that can produce required shifts in $R_{D^{(*)}}\left(R_{K^{(*)}}\right)$, one obtains $\Lambda_{\mathrm{NP}} \leq 1 \mathrm{TeV}\left(\Lambda_{\mathrm{NP}} \leq 30 \mathrm{TeV}\right)$ [8], where the operators in question scale as $\Lambda_{\mathrm{NP}}^{-2}$.

Over the last couple of years it has become clear that the most promising sources of new physics with regard to these lepton flavour universality violating hints are leptoquarks. These are the fields of either scalar or vector nature that couple quarks to leptons at the tree level. For a review on leptoquarks see, for example, Ref. [9]. The summary of those leptoquarks that can be used to address either one set or both sets of anomalies is presented in Table 1 [10]. Clearly, there is only one field, i.e., vector leptoquark $U_{1}(\mathbf{3}, \mathbf{1}, 2 / 3)$, that can simultaneously address both discrepancies [13], where the numbers in brackets represent the $U_{1}$ transformation properties under the SM gauge group $S U(3) \times S U(2) \times U(1)$. Note that scalar leptoquark $R_{2}(\mathbf{3}, \mathbf{2}, 7 / 6)$ can affect either $R_{D^{(*)}}$ or $R_{K^{(*)}}$ but it cannot possibly explain both sets of observables at the same time [10]. This then implies that one needs to use at least two scalar leptoquarks to generate viable new physics for $R_{D^{(*)}}$ and $R_{K^{(*)}}$. One such possibility is to use the $S_{1}(\overline{\mathbf{3}}, \mathbf{1}, 1 / 3)-S_{3}(\overline{\mathbf{3}}, \mathbf{3}, 1 / 3)$ combination [11, 12] whereas another option is to use the $R_{2}-S_{3}$ pair [14]. It is the physics of the latter combination that I pursue in the rest of this manuscript.

Concretely, I present an $S U(5)$ model with two light scalar leptoquarks, i.e., $R_{2}$ and $S_{3}$, that can address both sets of anomalies [14]. At low energies, $R_{2}$ generates a combination of scalar and tensor effective operators that accommodate $R_{D^{(*)}}$, while $S_{3}$ generates a $V-A$ operator which accommodates $R_{K^{(*)}}$. Since the Yukawa interactions have a common $S U(5)$ origin, both leptoquarks share one Yukawa coupling matrix. Also, if one takes into account all relevant flavour constraints, one finds that the preferred region in the parameter space is compatible with direct searches at the LHC. Furthermore, the perturbativity requirement for all the couplings up to the gauge coupling unification scale forces the masses of both $R_{2}$ and $S_{3}$ to be around $1 \mathrm{TeV}$.

\begin{tabular}{|c|c|c|c|}
\hline LEPTOQUARK & $R_{D^{(*)}}$ & $R_{K^{(*)}}$ & $R_{D^{(*)}} \& R_{K^{(*)}}$ \\
\hline \hline$S_{3}(\overline{\mathbf{3}}, \mathbf{3}, 1 / 3)$ & $\times$ & $\checkmark$ & $\times$ \\
$R_{2}(\mathbf{3}, \mathbf{2}, 7 / 6)$ & $\checkmark$ & $\checkmark$ & $\times$ \\
$S_{1}(\overline{\mathbf{3}}, \mathbf{1}, 1 / 3)$ & $\checkmark$ & $\times$ & $\times$ \\
\hline \hline$U_{3}(\mathbf{3}, \mathbf{3}, 2 / 3)$ & $\times$ & $\checkmark$ & $\times$ \\
$U_{1}(\mathbf{3}, \mathbf{1}, 2 / 3)$ & $\checkmark$ & $\checkmark$ & $\checkmark$ \\
\hline
\end{tabular}

Table 1: A list of potentially relevant leptoquarks for the $R_{D^{(*)}}$ and/or $R_{K^{(*)}}$ physics [10]. The numbers in brackets denote the leptoquark transformation properties under the SM gauge group $S U(3) \times S U(2) \times U(1)$. 


\section{The proposal}

The interactions of $R_{2}$ and $S_{3}$ with the SM fermions that are relevant for the $R_{D^{(*)}}$ and $R_{K^{(*)}}$ physics are

$$
\mathscr{L} \supset Y_{R}^{i j} \bar{Q}_{i}^{\prime} \ell_{R j}^{\prime} R_{2}+Y_{L}^{i j} \bar{u}_{R i}^{\prime} \widetilde{R}_{2}^{\dagger} L_{j}^{\prime}+Y^{i j} \bar{Q}_{i}^{\prime C} i \tau_{2}\left(\tau_{k} S_{3}^{k}\right) L_{j}^{\prime},
$$

where $Y_{R}, Y_{L}$, and $Y$ are Yukawa coupling matrices, $\tau_{k}$ denote the Pauli matrices, $S_{3}^{k}$ are the $S U(2)$ triplet components, $\widetilde{R_{2}} \equiv i \tau_{2} R_{2}^{*}$, and $i, j, k=1,2,3$. This part of the lagrangian, in the mass eigenstate basis, reads

$$
\begin{aligned}
\mathscr{L} \supset & +\left(V Y_{R} E_{R}^{\dagger}\right)^{i j} \bar{u}_{L i} \ell_{R j} R_{2}^{\frac{5}{3}}+\left(Y_{R} E_{R}^{\dagger}\right)^{i j} \bar{d}_{L i} \ell_{R j} R_{2}^{\frac{2}{3}}+\left(U_{R} Y_{L} U\right)^{i j} \bar{u}_{R i} v_{L j} R_{2}^{\frac{2}{3}}-\left(U_{R} Y_{L}\right)^{i j} \bar{u}_{R i} \ell_{L j} R_{2}^{\frac{5}{3}} \\
& -(Y U)^{i j} \bar{d}_{L i}^{C} \nu_{L j} S_{3}^{\frac{1}{3}}+2^{\frac{1}{2}}\left(V^{*} Y U\right)^{i j} \bar{u}_{L i}^{C} v_{L j} S_{3}^{-\frac{2}{3}}-2^{\frac{1}{2}} Y^{i j} \bar{d}_{L i}^{C} \ell_{L j} S_{3}^{\frac{4}{3}}-\left(V^{*} Y\right)^{i j} \bar{u}_{L i}^{C} \ell_{L j} S_{3}^{\frac{1}{3}},
\end{aligned}
$$

where $R_{2}^{(Q)}$ and $S_{3}^{(Q)}$ are the charge (and mass) eigenstates with charge $Q$. The mass eigenstates are defined via $u_{L, R}=U_{L, R} u_{L, R}^{\prime}, d_{L, R}=D_{L, R} d_{L, R}^{\prime}, \ell_{L, R}=E_{L, R} \ell_{L, R}^{\prime}$, and $v_{L}=N_{L} v_{L}^{\prime}$, where $U_{L, R}, D_{L, R}$, $E_{L, R}$, and $N_{L}$ are unitary matrices. Note that $V=U_{L} D_{L}^{\dagger} \equiv U_{L}$ and $U \equiv E_{L} N_{L}^{\dagger} \equiv N_{L}^{\dagger}$ are the CKM and PMNS matrices, respectively. I introduce the following properties for the Yukawa matrices

$$
Y_{R}=Y_{R}^{T}, \quad Y=-Y_{L},
$$

and assume

$$
Y_{R} E_{R}^{\dagger}=\left(\begin{array}{ccc}
0 & 0 & 0 \\
0 & 0 & 0 \\
0 & 0 & y_{R}^{b \tau}
\end{array}\right), U_{R} Y_{L}=\left(\begin{array}{ccc}
0 & 0 & 0 \\
0 & y_{L}^{c \mu} & y_{L}^{c \tau} \\
0 & 0 & 0
\end{array}\right),
$$

where $U_{R}^{22}=\cos \theta \equiv c_{\theta}, U_{R}^{23}=-\sin \theta \equiv-s_{\theta}$, and $\left|U_{R}^{11}\right|=1$. Hence, the only relevant new physics parameters for $R_{D^{(*)}}, R_{K^{(*)}}$, and all other flavour observables, are $m_{R_{2}}, m_{S_{3}}, y_{R}^{b \tau}, y_{L}^{c \mu}, y_{L}^{c \tau}$, and $\theta$.

In the simplest $S U(5)$ scenario that can accommodate light $R_{2}$ and $S_{3}$ and (re)produce associated flavour structure of Eq. (2.3), the scalar sector needs to contain $\underline{\mathbf{4 5}}$ and $\underline{\mathbf{5 0}}$ whereas the SM fermions comprise $\overline{\mathbf{5}}_{i}$ and $\mathbf{1 0}_{i}$, where $i(=1,2,3)$ is a generation index. Note that I denote $S U(5)$ representations with their dimensionalities, omit the $S U(5)$ indices in all expressions, and underline scalar representations throughout this manuscript.

To generate operators of Eq. (2.1) it is sufficient to introduce $a^{i j} \mathbf{1 0}_{i} \overline{\mathbf{5}}_{j} \overline{\mathbf{4 5}}$ and $b^{i j} \mathbf{1 0}_{i} \mathbf{1 0} j \underline{\mathbf{5 0}}$, where $a$ and $b\left(=b^{T}\right)$ are $3 \times 3$ matrices in the generation space. The former contraction couples $R_{2} \in \underline{\mathbf{4 5}}\left(S_{3} \in \underline{\mathbf{4 5}}\right)$ with the right-handed up-type quarks (quark doublets) and leptonic doublets, while the latter generates couplings of $R_{2} \in \underline{\mathbf{5 0}}$ with the quark doublets and right-handed charged leptons. To break $S U(5)$ down to the SM gauge group one can use $2 \mathbf{4}$ [15]. This allows one to write $m \underline{\mathbf{4 5}} \underline{\mathbf{5 0}} \underline{\mathbf{2 4}}$, where $m$ is a dimensionful parameter. The two $R_{2}$ 's that reside in $\underline{\mathbf{4 5}}$ and $\underline{\mathbf{5 0}}$ mix through this contraction allowing one to end up with two light scalars, i.e., $R_{2}$ and $S_{3}$, and one heavy $R_{2}$ state that completely decouples from the low-energy spectrum for large values of $m$. The relevant lagrangian, in the mass eigenstate basis of two light leptoquarks, is

$$
\begin{aligned}
\mathscr{L} \supset & +s_{\phi}\left(V b E_{R}^{\dagger}\right)^{i j} \bar{u}_{L i} \ell_{R j} R_{2}^{\frac{5}{3}}+s_{\phi}\left(b E_{R}^{\dagger}\right)^{i j} \bar{d}_{L i} \ell_{R j} R_{2}^{\frac{2}{3}}+c_{\phi}\left(U_{R} a U\right)^{i j} \bar{u}_{R i} v_{L j} R_{2}^{\frac{2}{3}}-c_{\phi}\left(U_{R} a\right)^{i j} \bar{u}_{R i} \ell_{L j} R_{2}^{\frac{5}{3}} \\
& +2^{-\frac{1}{2}}(a U)^{i j} \bar{d}_{L i}^{C} v_{L j} S_{3}^{\frac{1}{3}}-\left(V^{*} a U\right)^{i j} \bar{u}_{L i}^{C} v_{L j} S_{3}^{-\frac{2}{3}}+a^{i j} \bar{d}_{L i}^{C} \ell_{L j} S_{3}^{\frac{4}{3}}+2^{-\frac{1}{2}}\left(V^{*} a\right)^{i j} \bar{u}_{L i}^{C} \ell_{L j} S_{3}^{\frac{1}{3}},
\end{aligned}
$$


where I define the mixing angle between the two $R_{2}$ 's to be $\phi$. It is now trivial to compare Eq. (2.2) with Eq. (2.5) to obtain $Y=-a / \sqrt{2}, Y_{L}=c_{\phi} a$, and $Y_{R}=Y_{R}^{T}=s_{\phi} b$. The ansatz given in Eq. (2.3) is recovered by setting both $R_{2}$ states to mix maximally, i.e., $s_{\phi}=c_{\phi}=1 / \sqrt{2}$. Clearly, the two $S U$ (5) operators proportional to $a$ and $b$ suffice to generate three Yukawa matrices $Y, Y_{L}$, and $Y_{R}$.

To showcases the viability of the $S U(5)$ embedding I present in Fig. 1 a gauge coupling unification scenario for a full-fledged model that comprises $\mathbf{1 0}_{i}, \overline{\mathbf{5}}_{i}, \underline{\mathbf{5}}, \underline{\mathbf{2 4}}, \underline{\mathbf{4 5}}, \underline{\mathbf{5 0}}, i=1,2,3$. The masses of $R_{2}$ and $S_{3}$ are set at $m_{R_{2}}=800 \mathrm{GeV}$ and $m_{S_{3}}=2 \mathrm{TeV}$ to be in agreement with what is needed to address both $R_{D^{(*)}}$ and $R_{K^{(*)}}$ in a viable manner.

The scalar fields relevant for the gauge coupling unification considerations are $\Lambda_{a}(\mathbf{1}, \mathbf{2}, 1 / 2)$, $\Lambda_{b}(\mathbf{3}, \mathbf{1},-1 / 3) \in \underline{\mathbf{5}}, \phi_{a}(\mathbf{8}, \mathbf{1}, 0), \phi_{b}(\mathbf{1}, \mathbf{3}, 0) \in \underline{\mathbf{2 4}}, \Phi_{a}(\mathbf{8}, \mathbf{2}, 1 / 2), \Phi_{b}(\overline{\mathbf{6}}, \mathbf{1},-1 / 3), \Phi_{c}(\mathbf{3}, \mathbf{3},-1 / 3)$, $\Phi_{d}(\overline{\mathbf{3}}, \mathbf{2},-7 / 6), \Phi_{e}(\mathbf{3}, \mathbf{1},-1 / 3), \Phi_{f}(\overline{\mathbf{3}}, \mathbf{1}, 4 / 3), \Phi_{g}(\mathbf{1}, \mathbf{2}, 1 / 2) \in \underline{\mathbf{4 5}}$, and $\Omega_{a}(\mathbf{1}, \mathbf{1},-2), \Omega_{b}(\mathbf{3}, \mathbf{1},-1 / 3)$ $\Omega_{c}(\overline{\mathbf{3}}, \mathbf{2},-7 / 6), \Omega_{d}(\overline{\mathbf{6}}, \mathbf{3},-1 / 3), \Omega_{e}(\mathbf{6}, \mathbf{1}, 4 / 3), \Omega_{f}(\mathbf{8}, \mathbf{2}, 1 / 2) \in \underline{\mathbf{5 0}}$. Note, that one linear combination of $\Phi_{d}$ and $\Omega_{c}\left(\Lambda_{a}\right.$ and $\Phi_{g}$ ) yields light $R_{2}$ (the SM Higgs field) whereas the other combination resides at the unification scale $m_{\text {GUT }}$. Vertical lines in Fig. 1 represent masses of the scalar particles in the model whereas the unification scale $m_{\mathrm{GUT}}$ is identified with the mass of the proton decay mediating gauge bosons. To generate Fig. 1 I use $\alpha_{S}\left(M_{Z}\right)=0.1193, \alpha^{-1}\left(M_{Z}\right)=127.906$, and $\sin ^{2} \theta_{W}=0.23126$ [16] to maximise the unification scale for the case when $m_{R_{2}}=800 \mathrm{GeV}$ and $m_{S_{3}}=2 \mathrm{TeV}$ while the masses of all other fields are allowed to freely move between $1 \mathrm{TeV}$ and $m_{\mathrm{GUT}}$ except for $\Lambda_{b}, \Phi_{e}, \Phi_{f}$, and $\Omega_{b}$ that can mediate $d=6$ proton decay and that need to have masses at or above $3 \times 10^{11} \mathrm{GeV}[17,18]$.

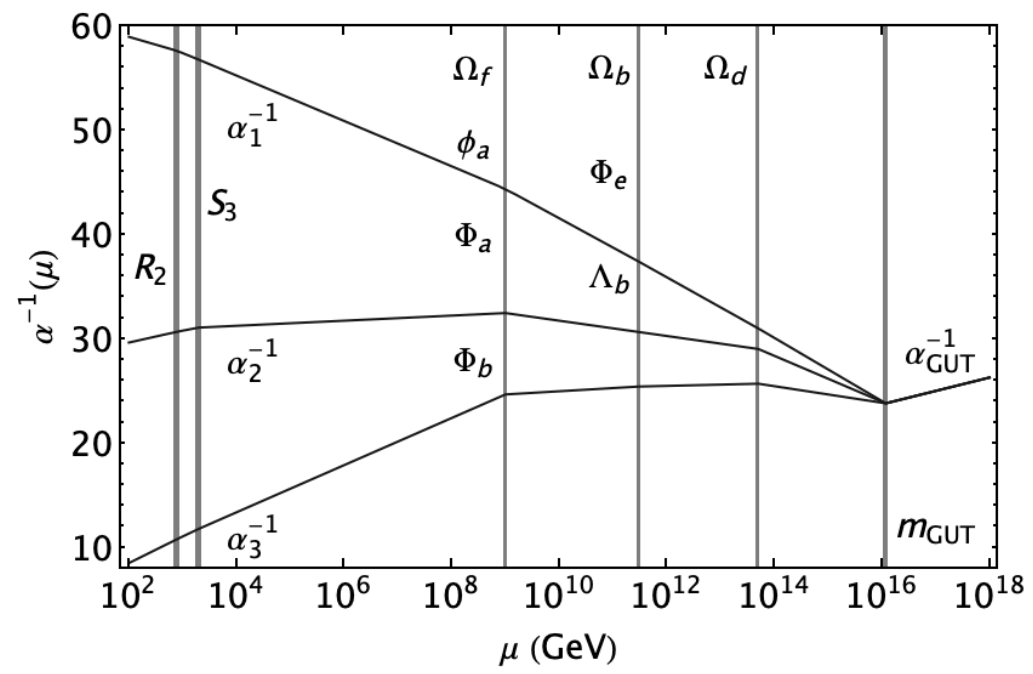

Figure 1: The gauge coupling unification for the $S U(5)$ model comprising $\mathbf{1 0}_{i}, \overline{\mathbf{5}}_{i}, \underline{\mathbf{5}}, \underline{\mathbf{2 4}}, \underline{\mathbf{4 5}}, \underline{\mathbf{5 0}}, i=1,2,3$.

I briefly outline the most prominent features of the flavour fit that is based on the lagrangian of Eq. (2.1) and the ansatz of Eqs. (2.3) and (2.4) in what follows and refer the reader for the detail analysis to Ref. [14]. The operators that are relevant for the $b \rightarrow c \tau \bar{\nu}(b \rightarrow s \mu \mu)$ transitions are

$$
\frac{y_{L}^{c \tau} y_{R}^{b \tau *}}{m_{R_{2}}^{2}}\left[\left(\bar{c}_{R} b_{L}\right)\left(\bar{\tau}_{R} v_{L}\right)+\frac{1}{4}\left(\bar{c}_{R} \sigma_{\mu \nu} b_{L}\right)\left(\bar{\tau}_{R} \sigma^{\mu v} v_{L}\right)\right] \quad\left(s_{2 \theta} \frac{\left|y_{L}^{c \mu}\right|^{2}}{m_{S_{3}}^{2}}\left(\bar{s}_{L} \gamma^{\mu} b_{L}\right)\left(\bar{\mu}_{L} \gamma_{\mu} v_{L}\right)\right)
$$


while the most important constraint originating from $\Delta m_{B_{s}}$ is generated by

$$
s_{2 \theta}^{2} \frac{\left[\left(y_{L}^{c \mu}\right)^{2}+\left(y_{L}^{c \tau}\right)^{2}\right]^{2}}{m_{S_{3}}^{2}}\left(\bar{s}_{L} \gamma^{\mu} b_{L}\right)^{2} .
$$

These considerations imply that, for small $s_{2 \theta}$, the effect of new physics in $b \rightarrow s \mu \mu$ is suppressed when compared to the impact on the $b \rightarrow c \tau \bar{v}$ transitions, whereas the $\Delta m_{B_{s}}$ contribution is doubly suppressed. This is what is phenomenologically preferred and the fit indeed yields $|\theta| \approx \pi / 2$ and $m_{R_{2}} \sim m_{S_{3}} \sim \Lambda_{\mathrm{NP}}$. I plot in Fig. 2 real and imaginary part of $g_{S}^{\tau}\left(\Lambda_{\mathrm{NP}}\right)=\left(y_{L}^{c \tau} y_{R}^{b \tau *}\right)\left(4 \sqrt{2} m_{R_{2}}^{2} G_{F} V_{c b}\right)$ to demonstrate the fit quality, where $\Lambda_{\mathrm{NP}} \approx 1 \mathrm{TeV}$. For more details on the the numerical analysis I, once again, refer the reader to Ref. [14].

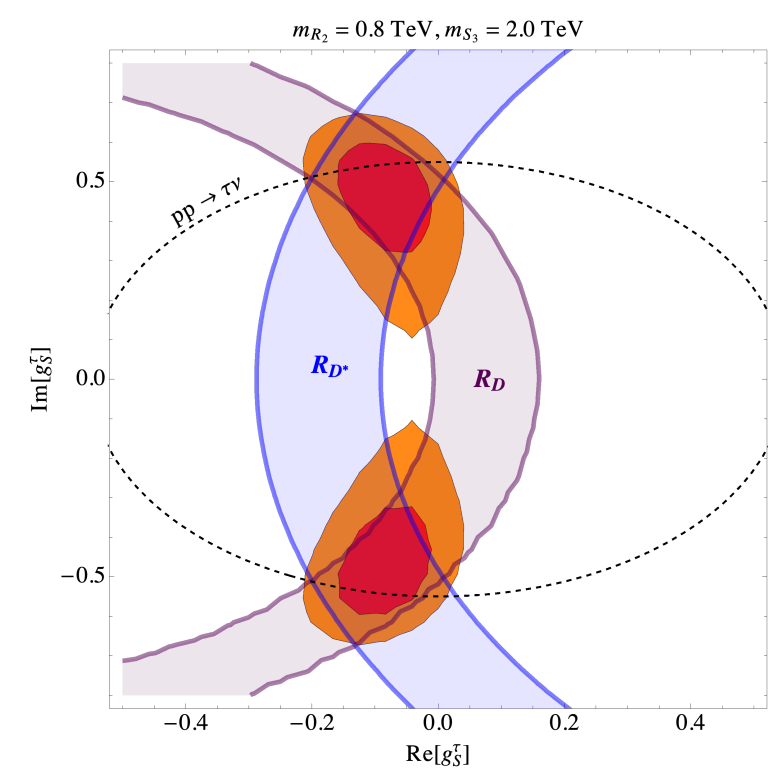

Figure 2: Results of the flavour fit in the $g_{S}^{\tau}$ plane for the transition $b \rightarrow c \tau \bar{\tau}_{\tau}$. The allowed $1 \sigma(2 \sigma)$ regions are rendered in red (orange). Separate constraints from $R_{D}$ and $R_{D^{*}}$ to $2 \sigma$ accuracy are shown by the blue and purple regions, respectively. The region outside of the dashed oval corresponds to the LHC exclusion [19].

Since the proposal calls for two light leptoquarks that couple to the SM fermions it is important to consider the existing and future LHC limits on the available parameter space of the model. I accordingly summarise the LHC exclusion potentials for the relevant processes at a projected luminosity $100 \mathrm{fb}^{-1}$ for $m_{R_{2}}=800 \mathrm{GeV}, m_{S_{3}}=2 \mathrm{TeV}$, and $|\theta| \approx \pi / 2$ in Fig. 3 .

An important prediction of this proposal is that $\mathscr{B}(B \rightarrow K \mu \tau)$ is bounded from above and below, as illustrated in Fig. 4, and is correlated with $\mathscr{B}\left(B \rightarrow K^{(*)} v v\right)$. This prediction can certainly be tested in the near future at Belle-II.

\section{Conclusions}

I describe a two scalar leptoquark extension of the SM that can accommodate the $R_{D^{(*)}}$ and $R_{K^{(*)}}$ discrepancies and related flavour observables, while being compatible with direct search constraints at the LHC. The extension has an $S U(5)$ origin that relates Yukawa couplings of the two 


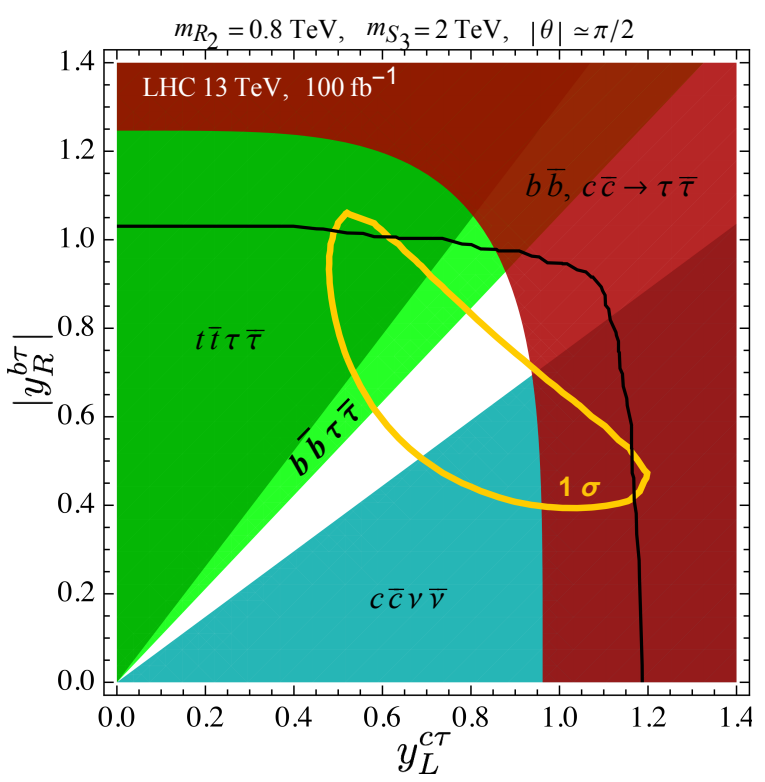

Figure 3: Summary of the LHC limits for relevant leptoquark processes at a projected luminosity of $100 \mathrm{fb}^{-1}$ for $m_{R_{2}}=800 \mathrm{GeV}, m_{S_{3}}=2 \mathrm{TeV}$, and $|\theta| \approx \pi / 2$. The red region corresponds to the exclusion limit from the high- $p_{T}$ di-tau search by ATLAS [20], while the green and turquoise exclusion regions come from the leptoquark pair production searches by CMS [21, 22, 23]. The region above the solid black contour represents values of the couplings that become non-perturbative at the unification scale. The region inside the yellow contour corresponds to the $1 \sigma$ fit to the low-energy observables.

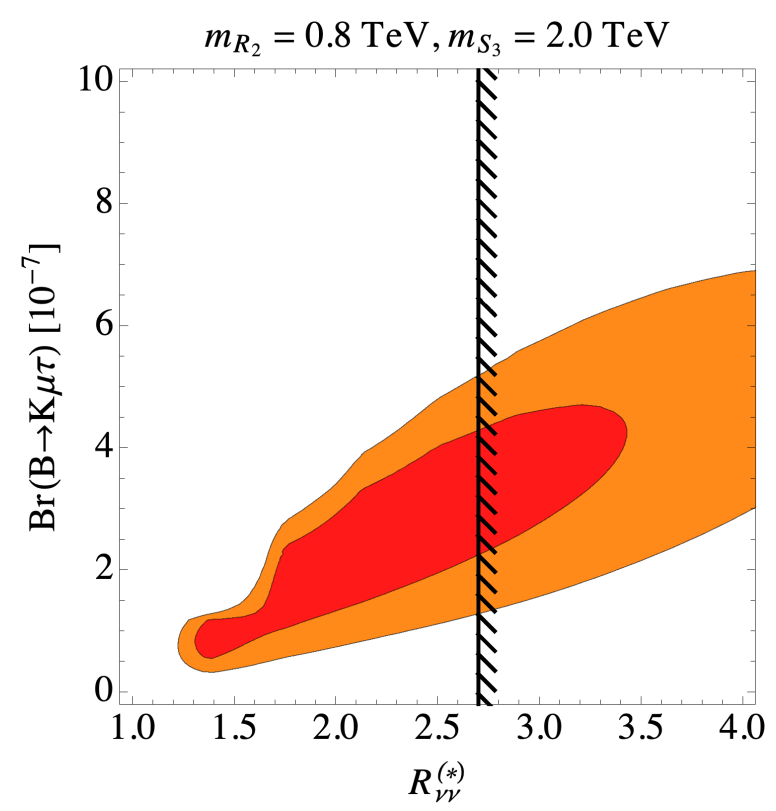

Figure 4: $\mathscr{B}(B \rightarrow K \mu \tau)$ vs. $R_{v v}=\mathscr{B}\left(B \rightarrow K^{(*)} v \bar{v}\right) / \mathscr{B}\left(B \rightarrow K^{(*)} v \bar{v}\right)^{\mathrm{SM}}$ for the $1 \sigma$ (red) and $2 \sigma$ (orange) regions of Fig. 1. The black line denotes the current experimental limit, $R_{v V}^{*}<2.7$ [24]. 
leptoquarks through a mixing angle and all Yukawas remain perturbative up to the unification scale. I provide prospects for future discoveries of the two light leptoquarks at the LHC and spell out predictions for several yet-to-be-measured flavour observables. In particular, the proposal predicts and correlates $\mathscr{B}(B \rightarrow K \mu \tau)$ with $\mathscr{B}\left(B \rightarrow K^{(*)} v v\right)$.

\section{References}

[1] C. Murgui et al., JHEP 1909 (2019) 103 [arXiv:1904.09311 [hep-ph]].

[2] R. X. Shi et al., arXiv:1905.08498 [hep-ph].

[3] M. Blanke et al., Phys. Rev. D 100 (2019) no.3, 035035 [arXiv:1905.08253 [hep-ph]].

[4] M. Algueró et al., Eur. Phys. J. C 79 (2019) no.8, 714 [arXiv:1903.09578 [hep-ph]].

[5] M. Ciuchini et al., Eur. Phys. J. C 79 (2019) no.8, 719 [arXiv:1903.09632 [hep-ph]].

[6] J. Aebischer et al., arXiv:1903.10434 [hep-ph].

[7] A. Arbey et al., Phys. Rev. D 100 (2019) no.1, 015045 [arXiv:1904.08399 [hep-ph]].

[8] L. Di Luzio and M. Nardecchia, Eur. Phys. J. C 77 (2017) no.8, 536 [arXiv:1706.01868 [hep-ph]].

[9] I. Doršner et al., Phys. Rept. 641 (2016) 1 [arXiv:1603.04993 [hep-ph]].

[10] A. Angelescu et al., JHEP 1810 (2018) 183 [arXiv:1808.08179 [hep-ph]].

[11] A. Crivellin et al., JHEP 1709 (2017) 040 [arXiv:1703.09226 [hep-ph]].

[12] D. Marzocca, JHEP 1807 (2018) 121 [arXiv:1803.10972 [hep-ph]].

[13] D. Buttazzo et al., JHEP 1711 (2017) 044 [arXiv:1706.07808 [hep-ph]].

[14] D. Bečirević et al., Phys. Rev. D 98 (2018) no.5, 055003 [arXiv:1806.05689 [hep-ph]].

[15] H. Georgi and S. L. Glashow, Phys. Rev. Lett. 32 (1974) 438.

[16] K. A. Olive et al. [Particle Data Group], Chin. Phys. C 38 (2014) 090001.

[17] I. Doršner, Phys. Rev. D 86 (2012) 055009 [arXiv:1206.5998 [hep-ph]].

[18] I. Doršner et al., Eur. Phys. J. C 77 (2017) no.6, 417 [arXiv:1701.08322 [hep-ph]].

[19] A. Greljo et al., Phys. Rev. Lett. 122 (2019) no.13, 131803 [arXiv:1811.07920 [hep-ph]].

[20] M. Aaboud et al. [ATLAS Collaboration], JHEP 1801 (2018) 055 [arXiv:1709.07242 [hep-ex]].

[21] A. M. Sirunyan et al. [CMS Collaboration], JHEP 1707 (2017) 121 [arXiv:1703.03995 [hep-ex]].

[22] CMS Collaboration [CMS Collaboration], CMS-PAS-SUS-16-036.

[23] A. M. Sirunyan et al. [CMS Collaboration], Eur. Phys. J. C 78 (2018) 707 [arXiv:1803.02864 [hep-ex]].

[24] J. Grygier et al. [Belle Collaboration], Phys. Rev. D 96 (2017) no.9, 091101 Addendum: [Phys. Rev. D 97 (2018) no.9, 099902] [arXiv:1702.03224 [hep-ex]]. 